\title{
FIXED POINT THEORY ON EXTENSION-TYPE SPACES AND ESSENTIAL MAPS ON TOPOLOGICAL SPACES
}

\author{
DONAL O'REGAN
}

Received 19 November 2003

We present several new fixed point results for admissible self-maps in extension-type spaces. We also discuss a continuation-type theorem for maps between topological spaces.

\section{Introduction}

In Section 2, we begin by presenting most of the up-to-date results in the literature [3, $5,6,7,8,12$ ] concerning fixed point theory in extension-type spaces. These results are then used to obtain a number of new fixed point theorems, one concerning approximate neighborhood extension spaces and another concerning inward-type maps in extensiontype spaces. Our first result was motivated by ideas in [12] whereas the second result is based on an argument of Ben-El-Mechaiekh and Kryszewski [9]. Also in Section 2 we present a new continuation theorem for maps defined between Hausdorff topological spaces, and our theorem improves results in [3].

For the remainder of this section we present some definitions and known results which will be needed throughout this paper. Suppose $X$ and $Y$ are topological spaces. Given a class $\mathscr{X}$ of maps, $\mathscr{X}(X, Y)$ denotes the set of maps $F: X \rightarrow 2^{Y}$ (nonempty subsets of $Y$ ) belonging to $\mathscr{X}$, and $\mathscr{X}_{c}$ the set of finite compositions of maps in $\mathscr{X}$. We let

$$
\mathscr{F}(\mathscr{X})=\{Z: \operatorname{Fix} F \neq \varnothing \forall F \in \mathscr{L}(Z, Z)\},
$$

where Fix $F$ denotes the set of fixed points of $F$.

The class $\mathscr{A}$ of maps is defined by the following properties:

(i) $\mathscr{A}$ contains the class $\mathscr{C}$ of single-valued continuous functions;

(ii) each $F \in \mathscr{A}_{c}$ is upper semicontinuous and closed valued;

(iii) $B^{n} \in \mathscr{F}\left(\mathscr{A}_{c}\right)$ for all $n \in\{1,2, \ldots\}$; here $B^{n}=\left\{x \in \mathbb{R}^{n}:\|x\| \leq 1\right\}$.

Remark 1.1. The class $\mathscr{A}$ is essentially due to Ben-El-Mechaiekh and Deguire [7]. It includes the class of maps $\mathcal{U}$ of Park ( $U$ is the class of maps defined by (i), (iii), and (iv) each $F \in U_{c}$ is upper semicontinuous and compact valued). Thus if each $F \in \mathscr{A}_{c}$ is compact 
valued, the classes $\mathscr{A}$ and $\mathscr{U}$ coincide and this is what occurs in Section 2 since our maps will be compact.

The following result can be found in [7, Proposition 2.2] (see also [11, page 286] for a special case).

Theorem 1.2. The Hilbert cube $I^{\infty}$ (subset of $l^{2}$ consisting of points $\left(x_{1}, x_{2}, \ldots\right)$ with $\left|x_{i}\right| \leq$ $1 / 2^{i}$ for all $i$ ) and the Tychonoff cube $T$ (Cartesian product of copies of the unit interval) are in $\mathscr{F}\left(\mathscr{A}_{c}\right)$.

We next consider the class $\mathcal{U}_{c}^{\kappa}(X, Y)$ (resp., $\mathscr{A}_{c}^{\kappa}(X, Y)$ ) of maps $F: X \rightarrow 2^{Y}$ such that for each $F$ and each nonempty compact subset $K$ of $X$, there exists a map $G \in \mathcal{U}_{c}(K, Y)$ (resp., $G \in \mathscr{A}_{c}(K, Y)$ ) such that $G(x) \subseteq F(x)$ for all $x \in K$.

Theorem 1.3. The Hilbert cube $I^{\infty}$ and the Tychonoff cube T are in $\mathscr{F}_{(}\left(\mathscr{A}_{c}^{\kappa}\right)$ (resp., $\left.\mathscr{F}_{(}\left(U_{c}^{\kappa}\right)\right)$.

Proof. Let $F \in \mathscr{A}_{c}^{\kappa}\left(I^{\infty}, I^{\infty}\right)$. We must show that Fix $F \neq \varnothing$. Now, by definition, there exists $G \in \mathscr{A}_{c}\left(I^{\infty}, I^{\infty}\right)$ with $G(x) \subseteq F(x)$ for all $x \in I^{\infty}$, so Theorem 1.2 guarantees that there exists $x \in I^{\infty}$ with $x \in G x$. In particular, $x \in F x$ so Fix $F \neq \varnothing$. Thus $I^{\infty} \in \mathscr{F}_{(}\left(\mathscr{A}_{c}^{\kappa}\right)$.

Notice that $U_{c}^{\kappa}$ is closed under compositions. To see this, let $X, Y$, and $Z$ be topological spaces, $F_{1} \in U_{c}^{\kappa}(X, Y), F_{2} \in U_{c}^{\kappa}(Y, Z)$, and $K$ a nonempty compact subset of $X$. Now there exists $G_{1} \in \mathcal{U}_{c}(K, Y)$ with $G_{1}(x) \subseteq F_{1}(x)$ for all $x \in K$. Also [4, page 464] guarantees that $G_{1}(K)$ is compact so there exists $G_{2} \in \mathcal{U}_{c}^{\kappa}\left(G_{1}(K), Z\right)$ with $G_{2}(y) \subseteq F_{2}(y)$ for all $y \in$ $G_{1}(K)$. As a result,

$$
G_{2} G_{1}(x) \subseteq F_{2} G_{1}(x) \subseteq F_{2} F_{1}(x) \quad \forall x \in K
$$

and $G_{2} G_{1} \in \mathcal{U}_{c}(X, Z)$.

For a subset $K$ of a topological space $X$, we denote by $\operatorname{Cov}_{X}(K)$ the set of all coverings of $K$ by open sets of $X$ (usually we write $\operatorname{Cov}(K)=\operatorname{Cov}_{X}(K)$ ). Given a map $F: X \rightarrow$ $2^{X}$ and $\alpha \in \operatorname{Cov}(X)$, a point $x \in X$ is said to be an $\alpha$-fixed point of $F$ if there exists a member $U \in \alpha$ such that $x \in U$ and $F(x) \cap U \neq \varnothing$. Given two maps $F, G: X \rightarrow 2^{Y}$ and $\alpha \in \operatorname{Cov}(Y), F$ and $G$ are said to be $\alpha$-close if for any $x \in X$ there exists $U_{x} \in \alpha, y \in$ $F(x) \cap U_{x}$, and $w \in G(x) \cap U_{x}$.

The following results can be found in [5, Lemmas 1.2 and 4.7].

Theorem 1.4. Let $X$ be a regular topological space and $F: X \rightarrow 2^{X}$ an upper semicontinuous map with closed values. Suppose there exists a cofinal family of coverings $\theta \subseteq \operatorname{Cov}_{X}(\overline{F(X)})$ such that $F$ has an $\alpha$-fixed point for every $\alpha \in \theta$. Then $F$ has a fixed point.

Theorem 1.5. Let T be a Tychonoff cube contained in a Hausdorff topological vector space. Then $T$ is a retract of $\operatorname{span}(T)$.

Remark 1.6. From Theorem 1.4 in proving the existence of fixed points in uniform spaces for upper semicontinuous compact maps with closed values, it suffices [6, page 298] to prove the existence of approximate fixed points (since open covers of a compact set $A$ 
admit refinements of the form $\{U[x]: x \in A\}$ where $U$ is a member of the uniformity [14, page 199], so such refinements form a cofinal family of open covers). Note also that uniform spaces are regular (in fact completely regular) [10, page 431] (see also [10, page $434]$ ). Note in Theorem 1.4 if $F$ is compact valued, then the assumption that $X$ is regular can be removed. For convenience in this paper we will apply Theorem 1.4 only when the space is uniform.

\section{Extension-type spaces}

We begin this section by recalling some results we established in [3]. By a space we mean a Hausdorff topological space. Let $Q$ be a class of topological spaces. A space $Y$ is an extension space for $Q$ (written $Y \in \mathrm{ES}(Q)$ ) if for all $X \in Q$ and all $K \subseteq X$ closed in $X$, any continuous function $f_{0}: K \rightarrow Y$ extends to a continuous function $f: X \rightarrow Y$.

Using (i) the fact that every compact space is homeomorphic to a closed subset of the Tychonoff cube and (ii) Theorem 1.3, we established the following result in [3].

Theorem 2.1. Let $X \in \mathrm{ES}$ (compact) and $F \in \mathcal{U}_{c}^{\kappa}(X, X)$ a compact map. Then $F$ has a fixed point.

Remark 2.2. If $X \in \mathrm{AR}$ (an absolute retract as defined in [11]), then of course $X \in$ ES(compact).

A space $Y$ is an approximate extension space for $Q$ (written $Y \in \operatorname{AES}(Q)$ ) if for all $\alpha \in \operatorname{Cov}(Y)$, all $X \in Q$, all $K \subseteq X$ closed in $X$, and any continuous function $f_{0}: K \rightarrow Y$, there exists a continuous function $f: X \rightarrow Y$ such that $\left.f\right|_{K}$ is $\alpha$-close to $f_{0}$.

Theorem 2.3. Let $X \in \mathrm{AES}$ (compact) be a uniform space and $F \in \mathcal{U}_{c}^{\kappa}(X, X)$ a compact upper semicontinuous map with closed values. Then $F$ has a fixed point.

Remark 2.4. This result was established in [3]. However, we excluded some assumptions ( $X$ uniform and $F$ upper semicontinuous with closed values) so the proof in [3] has to be adjusted slightly.

Proof. Let $\alpha \in \operatorname{Cov}_{X}(K)$ where $K=\overline{F(X)}$. From Theorem 1.4 (see Remark 1.6), it suffices to show that $F$ has an $\alpha$-fixed point. We know (see [13]) that $K$ can be embedded as a closed subset $K^{*}$ of $T$; let $s: K \rightarrow K^{*}$ be a homeomorphism. Also let $i: K \hookrightarrow X$ and $j: K^{*} \hookrightarrow T$ be inclusions. Next let $\alpha^{\prime}=\alpha \cup\{X \backslash K\}$ and note that $\alpha^{\prime}$ is an open covering of $X$. Let the continuous map $h: T \rightarrow X$ be such that $\left.h\right|_{K^{*}}$ and $s^{-1}$ are $\alpha^{\prime}$-close (guaranteed since $X \in \mathrm{AES}$ (compact)). Then it follows immediately from the definition (note that $\left.\alpha^{\prime}=\alpha \cup\{X \backslash K\}\right)$ that $h s: K \rightarrow X$ and $i: K \rightarrow X$ are $\alpha$-close. Let $G=j s F h$ and notice that $G \in \mathcal{U}_{c}^{\kappa}(T, T)$. Now Theorem 1.3 guarantees that there exists $x \in T$ with $x \in G x$. Let $y=h(x)$, and so, from the above, we have $y \in h j s F(y)$, that is, $y=h j s(q)$ for some $q \in F(y)$. Now since $h s$ and $i$ are $\alpha$-close, there exists $U \in \alpha$ with $h s(q) \in U$ and $i(q) \in U$, that is, $q \in U$ and $y=h j s(q)=h s(q) \in U$ since $s(q) \in K^{*}$. Thus $q \in U$ and $y \in U$, so $y \in U$ and $F(y) \cap U \neq \varnothing$ since $q \in F(y)$. As a result, $F$ has an $\alpha$-fixed point.

Definition 2.5. Let $V$ be a uniform space. Then $V$ is Schauder admissible if for every compact subset $K$ of $V$ and every covering $\alpha \in \operatorname{Cov}_{V}(K)$, there exists a continuous function (called the Schauder projection) $\pi_{\alpha}: K \rightarrow V$ such that 
(i) $\pi_{\alpha}$ and $i: K \hookrightarrow V$ are $\alpha$-close;

(ii) $\pi_{\alpha}(K)$ is contained in a subset $C \subseteq V$ with $C \in \operatorname{AES}$ (compact).

Theorem 2.6. Let $V$ be a uniform space and Schauder admissible and $F \in U_{c}^{\kappa}(V, V) a$ compact upper semicontinuous map with closed values. Then $F$ has a fixed point.

Proof. Let $K=\overline{F(X)}$ and let $\alpha \in \operatorname{Cov}_{V}(K)$. From Theorem 1.4 (see Remark 1.6), it suffices to show that $F$ has an $\alpha$-fixed point. There exists $\pi_{\alpha}: K \rightarrow V$ (as described in Definition 2.5) and a subset $C \subseteq V$ with $C \in \operatorname{AES}$ (compact) such that (here $F_{\alpha}=\pi_{\alpha} F$ )

$$
F_{\alpha}(V)=\pi_{\alpha} F(V) \subseteq C .
$$

Notice that $F_{\alpha} \in U_{c}^{\kappa}(C, C)$ is a compact upper semicontinuous map with closed (in fact compact) values. So Theorem 2.3 guarantees that there exists $x \in C$ with $x \in \pi_{\alpha} F(x)$, that is, $x=\pi_{\alpha} q$ for some $q \in F(x)$. Now Definition 2.5(i) guarantees that there exists $U \in \alpha$ with $\pi_{\alpha}(q) \in U$ and $i(q) \in U$, that is, $x \in U$ and $q \in U$. Thus $x \in U$ and $F(x) \cap U \neq \varnothing$ since $q \in F(x)$, so $F$ has an $\alpha$-fixed point.

A space $Y$ is a neighborhood extension space for $Q$ (written $Y \in \operatorname{NES}(Q)$ ) if for all $X \in Q$, all $K \subseteq X$ closed in $X$, and any continuous function $f_{0}: K \rightarrow Y$, there exists a continuous extension $f: U \rightarrow Y$ of $f_{0}$ over a neighborhood $U$ of $K$ in $X$.

Let $X \in \operatorname{NES}(Q)$ and $F \in \mathcal{U}_{c}^{\kappa}(X, X)$ a compact map. Now let $K, K^{*}, s$, and $i$ be as in the proof of Theorem 2.3. Let $U$ be an open neighborhood of $K^{*}$ in $T$ and let $h_{U}: U \rightarrow X$ be a continuous extension of $i s^{-1}: K^{*} \rightarrow X$ on $U$ (guaranteed since $X \in \mathrm{NES}$ (compact)). Let $j_{U}: K^{*} \hookrightarrow U$ be the natural embedding, so $h_{U} j_{U}=i s^{-1}$. Now consider $\operatorname{span}(T)$ in a Hausdorff locally convex topological vector space containing $T$. Now Theorem 1.5 guarantees that there exists a retraction $r: \operatorname{span}(T) \rightarrow T$. Let $i^{*}: U \hookrightarrow r^{-1}(U)$ be an inclusion and consider $G=i^{*} j_{U} s F h_{U} r$. Notice that $G \in \mathcal{U}_{c}^{\kappa}\left(r^{-1}(U), r^{-1}(U)\right)$. We now assume that

$$
G \in U_{c}^{\kappa}\left(r^{-1}(U), r^{-1}(U)\right) \text { has a fixed point. }
$$

Now there exists $x \in r^{-1}(U)$ with $x \in G x$. Let $y=h_{U} r(x)$, so $y \in h_{U} r i^{*} j_{U} s F(y)$, that is, $y=h_{U} r i^{*} j_{U} s(q)$ for some $q \in F(y)$. Since $h_{U}(z)=i s^{-1}(z)$ for $z \in K^{*}$, we have

$$
h_{U} r i^{*} j_{U} s(q)=\left(h_{U} r i^{*} j_{U}\right) s(q)=i(q)
$$

so $y \in F(y)$.

Theorem 2.7. Let $X \in \mathrm{NES}$ (compact) and $F \in \mathcal{U}_{c}^{\kappa}(X, X)$ a compact map. Also assume that (2.2) holds with $K, K^{*}, s, i, i^{*}, j_{U}, h_{U}$, and $r$ as described above. Then $F$ has a fixed point.

Remark 2.8. Theorem 2.7 was also established in [3]. Note that if $F$ is admissible in the sense of Gorniewicz and the Lefschetz set $\Lambda(F) \neq\{0\}$, then we know [11] that (2.2) holds. Note that if $X \in$ ANR (see [11]), then of course $X \in \mathrm{NES}$ (compact).

A space $Y$ is an approximate neighborhood extension space for $Q$ (written $Y \in \operatorname{ANES}(Q)$ ) if for all $\alpha \in \operatorname{Cov}(Y)$, all $X \in Q$, all $K \subseteq X$ closed in $X$, and any continuous function $f_{0}$ : $K \rightarrow Y$, there exists a neighborhood $U_{\alpha}$ of $K$ in $X$ and a continuous function $f_{\alpha}: U_{\alpha} \rightarrow Y$ such that $\left.f_{\alpha}\right|_{K}$ and $f_{0}$ are $\alpha$. 
Let $X \in$ ANES(compact) be a uniform space and $F \in U_{c}^{\kappa}(X, X)$ a compact upper semicontinuous map with closed values. Also let $\alpha \in \operatorname{Cov}_{X}(K)$ where $K=\overline{F(X)}$. To show that $F$ has a fixed point, it suffices (Theorem 1.4 and Remark 1.6) to show that $F$ has an $\alpha$-fixed point. Let $\alpha^{\prime}=\alpha \cup\{X \backslash K\}$ and let $K^{*}, s$, and $i$ be as in the proof of Theorem 2.3. Since $X \in$ ANES(compact), there exists an open neighborhood $U_{\alpha}$ of $K^{*}$ in $T$ and $f_{\alpha}: U_{\alpha} \rightarrow X$ a continuous function such that $\left.f_{\alpha}\right|_{K^{*}}$ and $s^{-1}$ are $\alpha^{\prime}$-close and as a result $f_{\alpha} s: K \rightarrow X$ and $i: K \rightarrow X$ are $\alpha$-close. Let $j_{U_{\alpha}}: K^{*} \hookrightarrow U_{\alpha}$ be the natural imbedding. We know (see [5, page 426]) that $U_{\alpha} \in \mathrm{NES}$ (compact). Also notice that $G_{\alpha}=j_{U_{\alpha}} s F f_{\alpha} \in U_{c}^{\kappa}\left(U_{\alpha}, U_{\alpha}\right)$ is a compact upper semicontinuous map with closed values. We now assume that

$$
G_{\alpha}=j_{U_{\alpha}} s F f_{\alpha} \in U_{c}^{\kappa}\left(U_{\alpha}, U_{\alpha}\right) \text { has a fixed point for each } \alpha \in \operatorname{Cov}_{X}(\overline{F(X)}) .
$$

We still have $\alpha \in \operatorname{Cov}_{X}(K)$ fixed and we let $x$ be a fixed point of $G_{\alpha}$. Now let $y_{\alpha}=f_{\alpha}(x)$, so $y=f_{\alpha} j_{U_{\alpha}} s F(y)$, that is, $y=f_{\alpha} j_{U_{\alpha}} s(q)$ for some $q \in F(y)$. Now since $f_{\alpha} s$ and $i$ are $\alpha$ close, there exists $U \in \alpha$ with $f_{\alpha} s(q) \in U$ and $i(q) \in U$, that is, $q \in U$ and $y=f_{\alpha} j_{U_{\alpha}} s(q)=$ $f_{\alpha} s(q) \in U$ since $s(q) \in K^{*}$. Thus $q \in U$ and $y \in U$, so

$$
y \in U, \quad F(y) \cap U \neq \varnothing \quad \text { since } q \in F(y) .
$$

Theorem 2.9. Let $X \in \mathrm{ANES}$ (compact) be a uniform space and $F \in \mathcal{U}_{c}^{\kappa}(X, X)$ a compact upper semicontinuous map with closed values. Also assume that (2.4) holds with $K, s, U_{\alpha}$, $j_{U_{\alpha}}$, and $f_{\alpha}$ as described above. Then $F$ has a fixed point.

Next we present continuation results for multimaps. Let $Y$ be a completely regular topological space and $U$ an open subset of $Y$. We consider a subclass $\mathscr{D}$ of $u_{c}^{\kappa}$. This subclass must have the following property: for subsets $X_{1}, X_{2}$, and $X_{3}$ of Hausdorff topological spaces, if $F \in \mathscr{D}\left(X_{2}, X_{3}\right)$ is compact and $f \in \mathscr{C}\left(X_{1}, X_{2}\right)$, then $F \circ f \in \mathscr{D}\left(X_{1}, X_{3}\right)$.

Definition 2.10. The map $F \in \mathscr{D}_{\partial U}(\bar{U}, Y)$ if $F \in \mathscr{D}(\bar{U}, Y)$ with $F$ compact and $x \notin F x$ for $x \in \partial U$; here $\bar{U}$ (resp., $\partial U$ ) denotes the closure (resp., the boundary) of $U$ in $Y$.

Definition 2.11. A map $F \in \mathscr{D}_{\partial U}(\bar{U}, Y)$ is essential in $\mathscr{D}_{\partial U}(\bar{U}, Y)$ if for every $G \in \mathscr{D}_{\partial U}(\bar{U}, Y)$ with $\left.G\right|_{\partial U}=\left.F\right|_{\partial U}$, there exists $x \in U$ with $x \in G x$.

Theorem 2.12 (homotopy invariance). Let $Y$ and $U$ be as above. Suppose $F \in \mathscr{D}_{\partial U}(\bar{U}, Y)$ is essential in $\mathscr{D}_{\partial U}(\bar{U}, Y)$ and $H \in \mathscr{D}(\bar{U} \times[0,1], Y)$ is a closed compact map with $H(x, 0)=$ $F(x)$ for $x \in \bar{U}$. Also assume that

$$
x \notin H_{t}(x) \quad \text { for any } x \in \partial U, t \in(0,1]\left(H_{t}(\cdot)=H(\cdot, t)\right) .
$$

Then $H_{1}$ has a fixed point in $U$.

Proof. Let

$$
B=\left\{x \in \bar{U}: x \in H_{t}(x) \text { for some } t \in[0,1]\right\} .
$$

When $t=0, H_{t}=F$, and since $F \in \mathscr{D}_{\partial U}(\bar{U}, Y)$ is essential in $\mathscr{D}_{\partial U}(\bar{U}, Y)$, there exists $x \in U$ with $x \in F x$. Thus $B \neq \varnothing$ and note that $B$ is closed, in fact compact (recall that $H$ is a closed, compact map). Notice also that (2.6) implies $B \cap \partial U=\varnothing$. Thus, since $Y$ is 
completely regular, there exists a continuous function $\mu: \bar{U} \rightarrow[0,1]$ with $\mu(\partial U)=0$ and $\mu(B)=1$. Define a map $R$ by $R(x)=H(x, \mu(x))$ for $x \in \bar{U}$. Let $j: \bar{U} \rightarrow \bar{U} \times[0,1]$ be given by $j(x)=(x, \mu(x))$. Note that $j$ is continuous, so $R=H \circ j \in \mathscr{D}(\bar{U}, Y)$ (see the description of the class $\mathscr{D}$ before Definition 2.10). In addition, $R$ is compact, and for $x \in \partial U$, we have $R(x)=H_{0}(x)=F(x)$. As a result, $R \in \mathscr{D}_{\partial U}(\bar{U}, Y)$ with $\left.R\right|_{\partial U}=\left.F\right|_{\partial U}$. Now since $F$ is essential in $\mathscr{D}_{\partial U}(\bar{U}, Y)$, there exists $x \in U$ with $x \in R(x)$, that is, $x \in H_{\mu(x)}(x)$. Thus $x \in B$ and so $\mu(x)=1$. Consequently, $x \in H_{1}(x)$.

Next we give an example of an essential map.

Theorem 2.13 (normalization). Let $Y$ and $U$ be as above with $0 \in U$. Suppose the following conditions are satisfied:

$$
\begin{gathered}
\text { for any map } \theta \in \mathscr{D}_{\partial U}(\bar{U}, Y) \text { with }\left.\theta\right|_{\partial U}=\{0\}, \text { the map } J \text { is in } u_{c}^{\kappa}(Y, Y) ; \\
J(x)= \begin{cases}\theta(x), & x \in \bar{U}, \\
\{0\}, & x \in Y \backslash \bar{U},\end{cases}
\end{gathered}
$$

and

$$
J \in U_{c}^{\kappa}(Y, Y) \text { has a fixed point. }
$$

Then the zero map is essential in $\mathscr{D}_{\partial U}(\bar{U}, Y)$.

Remark 2.14. Note that examples of spaces $Y$ for (2.9) to be true can be found in Theorems 2.1, 2.3, 2.6, 2.7, and 2.9 (notice that $J$ is compact).

Proof of Theorem 2.13. Let $\theta \in \mathscr{D}_{\partial U}(\bar{U}, Y)$ with $\left.\theta\right|_{\partial U}=\{0\}$. We must show that there exists $x \in U$ with $x \in \theta(x)$. Define a map $J$ as in (2.8). From (2.8) and (2.9), we know that there exists $x \in Y$ with $x \in J(x)$. Now if $x \notin U$, we have $x \in J(x)=\{0\}$, which is a contradiction since $0 \in U$. Thus $x \in U$ so $x \in J(x)=\theta(x)$.

Remark 2.15. Other homotopy and essential map results in a topological vector space setting can be found in $[1,2]$.

To conclude this paper, we discuss inward-type maps for a general class of admissible maps. The proof presented involves minor modifications of an argument due to BenEl-Mechaiekh and Kryszewski [9]. Let $Y$ be a normed space and $X \subseteq Y$, and consider a subclass $\mathscr{R}(X, Y)$ of $\boldsymbol{U}_{c}^{\kappa}(X, Y)$. This subclass must have the following properties: (i) if $X \subseteq Z \subseteq Y$ and if $I: X \hookrightarrow Z$ is an inclusion, $t>0$, and $F \in \mathscr{R}(X, Y)$ with $(I+t F)(X) \subseteq Z$, then $I+t F \in \mathcal{U}_{c}^{\kappa}(X, Z)$, and (ii) each $F \in \mathscr{R}(X, Y)$ is upper semicontinuous and compact valued.

In our next result we assume that $\Omega$ is a compact $\mathscr{L}$-retract [9], that is,

(A) $\Omega$ is a compact neighborhood retract of a normed space $E=(E,\|\cdot\|)$ and there exist $\beta>0, r: B(\Omega, \beta) \rightarrow \Omega$ a retraction, and $L>0$ such that $\|r(x)-x\| \leq L d(x ; \Omega)$ for $x \in B(\Omega, \beta)$. 
As a result,

$$
\exists \eta>0, \quad \eta<\frac{\beta}{2} \quad \text { with }\|r(x)-x\|<\eta \forall x \in B(\Omega, \eta) .
$$

Theorem 2.16. Let $E=(E,\|\cdot\|)$ be a normed space and $\Omega$ as in assumption $(A)$, and assume either ( $i) \Omega$ is Schauder admissible or (ii) (2.2) holds with $X=\Omega$. In addition, suppose $F \in \mathscr{R}(\Omega, E)$ with

$$
F(x) \subseteq C_{\Omega}(x) \quad \forall x \in \Omega
$$

Then there exists $x \in \Omega$ with $0 \in F x$.

Remark 2.17. Here $C_{\Omega}$ is the Clarke tangent cone, that is,

$$
C_{\Omega}(x)=\{v \in E: c(x, v)=0\},
$$

where

$$
c(x, y)=\limsup _{\substack{y \rightarrow x, y \in \Omega \\ t \downarrow 0}} \frac{d(x+t v ; \Omega)}{t} .
$$

Remark 2.18. If $\Omega$ is a compact neighborhood retract, then of course $\Omega \in$ NES(compact).

Remark 2.19. The proof is basically due to Ben-El-Mechaiekh and Kryszewski [9] and is based on [9, Lemma 5.1] (this lemma is a modification of a standard argument in the literature using partitions of unity).

Proof. Now [9, Lemma 5.1] (choose $\Psi(x)=\{x \in E: c(x, v)<\delta\}(\delta>0$ appropriately chosen), $\Phi(x)=c o(F(x))$ and apply the argument in [9, page 4176]) implies that there exists $M>0$ such that for each $x \in K$ and each $y \in F x$, we have $\|y\| \leq M$. Choose $\tau>0$ with $M \tau<\eta$ (here $\eta$ is as in (2.10)) and a sequence $\left(t_{n}\right)_{n \in N}$ in $(0, \tau]$ with $t_{n} \downarrow 0$; here $N=\{1,2, \ldots\}$. Define a sequence of maps $\psi_{n}, n \in N$, by

$$
\psi_{n}(x)=r\left(x+t_{n} F(x)\right) \quad \text { for } x \in \Omega \text {; }
$$

note that $d\left(x+t_{n} y ; \Omega\right)<\eta$ for $x \in \Omega$ and $y \in F(x)$ since $M \tau<\eta$. Fix $n \in N$ and notice that $\psi_{n} \in U_{c}^{\kappa}(\Omega, \Omega)$ is a compact map (note that $\Omega$ is compact and $\psi_{n}$ is upper semicontinuous with compact values). Now Theorem 2.6 or Theorem 2.7 guarantees that there exists $x_{n} \in \Omega$ and $y_{n} \in F x_{n}$ with

$$
x_{n}=r\left(x_{n}+t_{n} y_{n}\right)
$$

Also notice from (2.15) and assumption (A) (note that $M \tau<\eta<\beta / 2<\beta$ ) that

$$
t_{n}\left\|y_{n}\right\|=\left\|x_{n}+t_{n} y_{n}-r\left(x_{n}+t_{n} y_{n}\right)\right\| \leq L d\left(x_{n}+t_{n} y_{n} ; \Omega\right) \text {. }
$$

Now $\Omega$ is compact so $F(\Omega)$ is compact, and as a result, there exists a subsequence $S$ of $N$ with $\left(x_{n}, y_{n}\right) \in \operatorname{Graph} F$ and $\left(x_{n}, y_{n}\right) \rightarrow(\bar{x}, \bar{y})$ as $n \rightarrow \infty$ in $S$. Of course, since $F$ is upper 
semicontinuous, we have $\bar{y} \in F(\bar{x})$. Also from (2.11), we have $F(\bar{x}) \subseteq C_{\Omega}(\bar{x})$ and as a result, $\bar{y} \in F(\bar{x}) \subseteq C_{\Omega}(\bar{x})$, so $c(\bar{x}, \bar{y})=0$. Note also that

$$
d\left(x_{n}+t_{n} y_{n} ; \Omega\right) \leq d\left(x_{n}+t_{n} \bar{y} ; \Omega\right)+t_{n}\left\|y_{n}-\bar{y}\right\|
$$

and this together with (2.16) yields

$$
\|\bar{y}\|=\limsup _{n \rightarrow \infty}\left\|y_{n}\right\| \leq \limsup \left(\frac{\operatorname{Ld}\left(x_{n}+t_{n} \bar{y} ; \Omega\right)}{t_{n}}+\left\|y_{n}-\bar{y}\right\|\right)=c(\bar{x}, \bar{y})=0,
$$

so $0 \in F(\bar{x})$.

\section{References}

[1] R. P. Agarwal and D. O'Regan, Homotopy and Leray-Schauder principles for multi maps, Nonlinear Anal. Forum 7 (2002), no. 1, 103-111.

[2] - An essential map theory for $\mathcal{U}_{c}^{\kappa}$ and PK maps, Topol. Methods Nonlinear Anal. 21 (2003), no. 2, 375-386.

[3] R. P. Agarwal, D. O'Regan, and S. Park, Fixed point theory for multimaps in extension type spaces, J. Korean Math. Soc. 39 (2002), no. 4, 579-591.

[4] C. D. Aliprantis and K. C. Border, Infinite-Dimensional Analysis, Studies in Economic Theory, vol. 4, Springer-Verlag, Berlin, 1994.

[5] H. Ben-El-Mechaiekh, The coincidence problem for compositions of set-valued maps, Bull. Austral. Math. Soc. 41 (1990), no. 3, 421-434.

[6] Spaces and maps approximation and fixed points, J. Comput. Appl. Math. 113 (2000), no. 1-2, 283-308.

[7] H. Ben-El-Mechaiekh and P. Deguire, General fixed point theorems for nonconvex set-valued maps, C. R. Acad. Sci. Paris Sér. I Math. 312 (1991), no. 6, 433-438.

[8] Approachability and fixed points for nonconvex set-valued maps, J. Math. Anal. Appl. 170 (1992), no. 2, 477-500.

[9] H. Ben-El-Mechaiekh and W. Kryszewski, Equilibria of set-valued maps on nonconvex domains, Trans. Amer. Math. Soc. 349 (1997), no. 10, 4159-4179.

[10] R. Engelking, General Topology, Sigma Series in Pure Mathematics, vol. 6, Heldermann Verlag, Berlin, 1989.

[11] L. Górniewicz, Topological Fixed Point Theory of Multivalued Mappings, Mathematics and Its Applications, vol. 495, Kluwer Academic Publishers, Dordrecht, 1999.

[12] A. Granas, Fixed point theorems for the approximative ANR-s, Bull. Acad. Polon. Sci. Sér. Sci. Math. Astronom. Phys. 16 (1967), 15-19.

[13] _ Points Fixes pour les Applications Compactes: Espaces de Lefschetz et la Théorie de l'Indice, Séminaire de Mathématiques Supérieures, vol. 68, Presses de l'Université de Montréal, Montreal, 1980.

[14] J. L. Kelley, General Topology, D. Van Nostrand, New York, 1955.

Donal O'Regan: Department of Mathematics, National University of Ireland, Galway, Ireland E-mail address: donal.oregan@nuigalway.ie 